\title{
On Strongly Stable Approximations
}

\author{
F. ARÁNDIGA and V. CASELLES ${ }^{1}$
}

\begin{abstract}
In this paper we prove that the convergence of $\left(T-T_{n}\right) T_{n}^{k}$ to zero in operator norm (plus some technical conditions) is a sufficient condition for $T_{n}$ to be a strongly stable approximation to $T$, thus extending some previous results existing in the literature.
\end{abstract}

\section{INTRODUCTION}

Let $T$ be a bounded linear operator on a complex Banach space $X$ and $\lambda$ be an isolated nonzero eigenvalue of $T$ with finite algebraic multiplicity. Let $T_{n}$ be a sequence of bounded linear operators on $X$. In this paper we give sufficient conditions under which $T_{n}$ is a strongly stable approximation of $T$ at $\lambda$. This work is motivated by recent results of M. Ahues [1], R. Bouldin [4] and M. Thambar Nair [10]. Our effort is directed to establish a minimal set of assumptions to guarantee that $T_{n}$ is a strongly stable approximation of $T$ and $\lambda$ in the spirit of the above mentioned works.

\footnotetext{
1 Partially supported by D.G.I.C.Y.T. project PS90-0271. 1991 Mathematics Subject Classification: 65 F15, $15 \mathrm{~A} 18$

Editorial Complutense. Madrid, 1994.
} 


\section{PRELIMINARIES AND STATEMENT OF THE MAIN RESULT}

In the sequel let $X$ be a complex Banach space and let $\mathcal{L}(X)$ be the space of bounded linear operators on $X$. The spectrum of $T$, i.e. the set of $z \in C$ such that $z I-T$ is not invertible in $\mathcal{L}(X)$ will be denoted by $\sigma(T)$. If $z \in \rho(T):=C \sim \sigma(T)$, the resolvent set, $R(z, T):=$ $(z I-T)^{-1} \in \mathcal{L}(X)$.

$\lambda \in \sigma(T)$ is a Riesz point of $\sigma(T)$ if $\lambda$ is a pole of the resolvent $R\left(z, T\right.$ ) with a residuum $P=\frac{1}{2 \pi i} \int_{\Gamma} R(z, T) d z$ of finite rank ( $\Gamma$ is a curve on the complex plane around $\lambda$ containing $\lambda$ as the only singularity of $R(z, T))$. The Riesz points of $\sigma(T)$ are the isolated eigenvalues of $T$ with finite algebraic multiplicity. If $\lambda \in \sigma(T)$ is not a Riesz point of $\sigma(T)$, we write $\lambda \in \sigma_{e s s}(T)$.

Let us explain an ultrapower construction introduced by $S$. Berberian which is used in spectral theory ([9] V.1) Let us fix an ultrafilter $\mathcal{U}$ on $\mathbf{N}$ contajning the Frechet filter. The ultrapower of $X$ with respect to $\mathcal{U}$, denoted by $\widehat{X}_{U}$ or simply by $\hat{X}$ is defined by $\ell^{\infty}(X) / C_{\mathcal{U}}(X)$ where

$$
\ell^{\infty}(X):=\left\{\left(x_{n}\right)_{u}: x_{n} \in X, \sup _{n}\left\|x_{n}\right\|<+\infty\right\}
$$

and

$$
C_{U}:=\left\{\left(x_{n}\right) \in \ell^{\infty}(X): \lim _{\mathcal{U}}\left\|x_{n}\right\|=0\right\}
$$

Operators on $X$ can be lifted to operators on $\widehat{X}$ by $\widehat{T} \widehat{x}=\left(T x_{n}\right)_{\mathcal{U}}$, $\widehat{x} \in \hat{X}, T \in \mathcal{L}(X)$ in such a way that $\sigma(\widehat{T})=\sigma(T)$, ([9], Theorem V.1.4). The approximate point spectrum of $T$ is converted into the point spectrum of $\widehat{T}([9]$, Theorem. V.1.4). Now, we recall the following result from [6].

Theorem 2.1. Let $T \in \mathcal{L}(X)$. Let $\partial_{\infty} \sigma(T)$ the exterior boundary of $\sigma(T)$ (= the boundary of the unbounded connected component of $\rho(T)$ ). Then $\partial_{\infty} \sigma(T) \cap \sigma_{e s s}(T)=\partial_{\infty} \sigma(T) \cap\{z \in C: \operatorname{dim} \operatorname{Ker}(z-\widehat{T})$ is infinite $\}$.

This result means that the eigenspace associated to a Riesz point on the exterior boundary of $\sigma(T)$ is contained in $X$ and cannot be enlarged by going to $\widehat{X}$. 
The following definitions are taken from [7].

Definition 2.1. Let $T, T_{n} \in \mathcal{L}(X) n=1,2, \ldots$ The stable convergence of $T_{n}$ to $T$, denoted by $T_{n} \stackrel{s}{\rightarrow} T$, means that:

1. $T_{n} x \rightarrow T x$ in $X$ for all $x \in X$

2. $\exists M>0, \exists N \in \mathbf{N}$ such that $\forall n \geq N, T_{n}^{-1} \in \mathcal{L}(X)$ and $\left\|T_{n}^{-1}\right\| \leq$ $M$.

Definition 2.2. Let $T, T_{n} \in \mathcal{L}(X) n=1,2, \ldots$ Let $\lambda$ be a Riesz point of $\sigma(T)$ of algebraic multiplicity $m$. We say that $T_{n}$ is strongly stable convergent to $T$ at $\lambda$ and denote it by $T_{n} \stackrel{\text { ss }}{\longrightarrow} T$ at $\lambda$ if there exists a closed Jordan curve $\Gamma \subseteq \rho(T)$ around $\lambda$ such that $\lambda$ is the only spectral point of $T$ inside $\Gamma$ and such that

1. $T_{n}-z \stackrel{s}{\longrightarrow} T-z$, for all $z \in \Gamma$

2. $\operatorname{dim} P_{n} X=m$ for $n$ large enough, where $P_{n}$ is the spectral projection associated with $\sigma\left(T_{n}\right) \cap \Delta$ ( $\Delta$ denotes the set of points $z \in C$ inside $\Gamma$ ).

Here one should mention that if $T_{n}-z \stackrel{s}{\longrightarrow} T-z, \forall z \in \Gamma$, then for some $N=N(\Gamma) \in \mathbf{N}$ and $n \geq N, \sigma\left(T_{n}\right) \cap \Delta$ is an spectral set of $\sigma\left(T_{n}\right)$ and we may define $P_{n}=\frac{1}{2 \pi i} \int_{\Gamma} R\left(z, T_{n}\right) d z([7] \mathrm{p} .231,232)$.

The strong stability of $T_{n}$ at $\lambda$ is a sufficient condition for the convergence of the eigenvalues of $T_{n}$ inside $\Gamma$ with preservation of the algebraic multiplicities ([7] Theorem 6.7).

Let us recall the results of M. Ahues [1], R. Bouldin [4] and M. Thambar Nair [10] mentioned in the introduction.

Let $T, T_{n} \in \mathcal{L}(X)$ and let us suppose that

(I) $T_{n} x \longrightarrow T x$ for all $x \in X$.

(II) $\left\|\left(T-T_{n_{n}}\right) T_{n}\right\| \longrightarrow 0$ as $n \longrightarrow+\infty$.

In [1], Ahues proved that if $T \in \mathcal{L}(X)$ is a compact operator satisfying (I) and (II) and $\lambda \neq 0$ is an eigenvalue of $T$, then $T_{n} \stackrel{s s}{\rightarrow} T$ at $\lambda$. 
Then R. Bouldin [4] eliminated the compacness of $T$ supposing that $\lambda \neq 0$ is a Riesz point of $\sigma(T)$ and adding the assumption

(III) $\left\|T_{n}\left(T-T_{n}\right)\right\| \longrightarrow 0$ si $n \longrightarrow+\infty$.

Later, M. Thambar Nair [10] proved that if $T$ and $T_{n} \in \mathcal{L}(X)$ satisfy (I), (II), $\lambda \neq 0$ is a Riesz point of $\sigma(T)$ and one of the following assumptions

(IV) $\left\|\left(T-T_{n}\right) T\right\| \longrightarrow 0$

(V) $\left\|T_{n}\left(T-T_{n}\right)\right\| \longrightarrow 0$

(VI) $\exists N \in \mathbf{N}$ such that $\forall n \geq N T_{n}$ is compact

(VII) $\exists N \in \mathbf{N}$ such that $\forall n \geq N T-T_{n}$ is compact holds, then $T_{n} \stackrel{s s}{\longrightarrow} T$ at $\lambda$.

Our purpose is to prove the following result.

Theorem 2.2. Let $T, T_{n} \in \mathcal{L}(X), n=1,2, \ldots$ be such that:

1. $T_{n} x \longrightarrow T x$ for all $x \in X$

2. $\left\|\left(T-T_{n}\right) T_{n}^{k}\right\| \longrightarrow 0$ if $n \longrightarrow+\infty$, for some $k \in \mathbf{N}$

and let $\lambda \in \partial_{\infty} \sigma(T), \lambda \neq 0$, be a Riesz point of $\sigma(T)$. Then, $T_{n} \stackrel{s s}{\longrightarrow} T$ at $\lambda$.

Thus, the assumption that $\lambda \in \partial_{\infty} \sigma(T)$ (which we believe of no harm in practice), permits us to economize the set of assumptions in the previous results.

An interesting consequence of Theorem 2.2 is:

Theorem 2.3. Let $T, T_{n} \in \mathcal{L}(X), n=1,2, \ldots$ be such that:

1. $T_{n} x \longrightarrow$ Tx for all $x \in X$

2. $\left\|\left(T-T_{n}\right) T_{n}^{k}\right\| \longrightarrow 0$ as $n \longrightarrow+\infty$, for some $k \in \mathbf{N}$

and let $\lambda \in \partial_{\infty} \sigma(T), \lambda \neq 0$, be a Riesz point of $\sigma(T)$ of algebraic multiplicity $m$ and index $\nu \leq m$. Then, for some circle $\Gamma$ of radius $r>0$ around $\lambda$, there are exactly $m$ eigenvalues of $T_{n}$ inside $\Gamma$ (counted 
according to their multiplicities) and if $\sigma_{n}=\{z \in \sigma(T):|z-\lambda|<r\}$, then

$$
\max _{z \in \sigma_{n}}|z-\lambda|^{\nu} \leq C\left\|T_{n}-T\right\|_{X(T, \lambda)}
$$

where $X(T, \lambda)$ is the spectral subspace of $X$ associated to $\lambda$ and $\|\cdot\|_{X(T, \lambda)}$ denotes the operator norm restricted to $X(T, \lambda)$.

Now, we go directly into the proof of Theorem 2.2 .

\section{PROOF OF THE MAIN RESULT}

First of all we recall the following result proved by $\mathrm{M}$. Thamban Nair [10]. We include its proof here for the sake of completeness.

Proposition 3.1. Let $T, T_{n} \in \mathcal{L}(X), n=1,2, \ldots$ and let $\lambda \neq 0$ be a Riesz point of $\sigma(T)$. Let $\Gamma$ be a closed Jordan curve around $\lambda$ separating $\lambda$ from $\sigma(T) \sim\{\lambda\}$. Suppose that:

1. $T_{n} x \longrightarrow T x$ for all $x \in X$

2. $\left\|\left(T-T_{n}\right) T_{n}^{k}\right\| \longrightarrow 0$ as $n \longrightarrow+\infty$, for some $k \in \mathbf{N}$

3. $T_{n}-z \stackrel{s}{\longrightarrow} T-z \quad \forall z \in \Gamma$.

Then $T_{n} \stackrel{s s}{\longrightarrow} T$ at $\lambda$.

Proof. Let

$$
P=\frac{1}{2 \pi i} \int_{\Gamma} R(z, T) d z
$$

and for some $N=N(\Gamma)$ and all $n \geq N$,

$$
P_{n}=\frac{1}{2 \pi i} \int_{\Gamma} R\left(z, T_{n}\right) d z
$$

The result will follow if we prove that $\left\|\left(P-P_{n}\right) P\right\| \longrightarrow 0$ and $\left\|\left(P-P_{n}\right) P_{n}\right\| \longrightarrow 0$.

Since

$$
R(z, T)-R\left(z, T_{n}\right)=R\left(z, T_{n}\right)\left(T-T_{n}\right) R(z, T)=
$$




$$
=R(z, T)\left(T-T_{n}\right) R\left(z, T_{n}\right),
$$

then

$$
\begin{gathered}
\left(P-P_{n}\right) P=\frac{1}{2 \pi i} \int_{\Gamma} R\left(z, T_{n}\right)\left(T-T_{n}\right) R(z, T) P d z \\
\left(P-P_{n}\right) P_{n}=\frac{1}{2 \pi i} \int_{\Gamma} R(z, T)\left(T-T_{n}\right) R\left(z, T_{n}\right) P_{n} d z
\end{gathered}
$$

Since $T_{n}-z \stackrel{s}{\longrightarrow} T-z \forall z \in \Gamma$, then for some $N=N(\Gamma) \in \mathbf{N}$

$$
\sup _{\substack{z \in \Gamma \\ n \geq N}}\left\|R\left(z, T_{n}\right)\right\|<+\infty
$$

([7], Cor. 5.2). It follows that for some $M>0,\left\|\left(P-P_{n}\right) P\right\| \leq$ $M\left\|\left(T-T_{n}\right) P\right\|$. Now since $T_{n} x \longrightarrow T x$ for all $x \in X$ and $P$ is a projection of finite rank, $\left\|\left(T-T_{n}\right) P\right\| \longrightarrow 0$. Hence $\left\|\left(P-P_{n}\right) P\right\| \longrightarrow 0$. In the same way, $\left\|\left(P-P_{n}\right) P_{n}\right\| \leq M\left\|\left(T-T_{n}\right) P_{n}\right\|$ for some $M>0$. Now

$$
\left(T-T_{n}\right) P_{n}=\frac{1}{2 \pi i} \int_{\Gamma}\left(T-T_{n}\right) R\left(z, T_{n}\right) d z
$$

and using $T_{n} R\left(z, T_{n}\right)=z R\left(z, T_{n}\right)-I$,

$$
\left(T-T_{n}\right) P_{n}=\frac{1}{2 \pi i} \int_{\Gamma}\left(T-T_{n}\right)\left(T_{n} R\left(z, T_{n}\right)+I\right) \frac{d z}{z} .
$$

Since $\lambda \neq 0$, we may suppose without loss of generality that 0 is not inside $\Gamma$. Then $\int_{\Gamma} \frac{d z}{z}=0$ and

$$
\left(T-T_{n}\right) P_{n}=\frac{1}{2 \pi i} \int_{\Gamma}\left(T-T_{n}\right) T_{n} R\left(z, T_{n}\right) \frac{d z}{z} .
$$

Proceeding inductively,

$$
\left(T-T_{n}\right) P_{n}=\frac{1}{2 \pi i} \int_{\Gamma}\left(T-T_{n}\right) T_{n}^{k} R\left(z, T_{n}\right) \frac{d z}{z^{k}} .
$$


Using assumption (2) and (1) it follows that $\left\|\left(T-T_{n}\right) P_{n}\right\| \longrightarrow 0$. Hence $\left\|\left(P-P_{n}\right) P_{n}\right\| \longrightarrow 0$. The proof follows in a standard way: it is easy to see that the gap between $X_{n}=P_{n} X$ and $X_{0}=P X$ ([8],p. 197 for the definition of gap), denoted by $\gamma\left(X_{n}, X_{0}\right)$ does not exceed

$$
\max \left\{\left\|\left(P-P_{n}\right) P\right\|,\left\|\left(P-P_{n}\right) P_{n}\right\|\right\}
$$

From $\left\|\left(P_{n}-P\right) P\right\|,\left\|\left(P_{n}-P\right) P_{n}\right\| \longrightarrow 0$ it follows that $\gamma\left(X_{n}, X_{0}\right)<1$ for $n$ large enough. According to ([8], p. 200 Cor. 2.6) this implies that $\operatorname{dim} X_{n}=\operatorname{dim} X_{0}$ and the proposition is proved (see [4], p. 8).

Lemma 3.1. Let $T, T_{n}, n=1,2, \ldots$ and $\lambda$ be as in Theorem 2.2. Let $\lambda_{n} \in \partial_{\infty} \sigma\left(T_{n}\right)$ be such that $\lambda_{n} \longrightarrow \lambda$. Then, there exists $n_{0} \in \mathbf{N}$ such that $\lambda_{n}$ is a Riesz point of $\sigma\left(T_{n}\right), \forall n \geq n_{0}$.

Proof. Let $F_{n}=\operatorname{Ker}\left(\lambda_{n}-\widehat{T}_{n}\right)$. Suppose that $\operatorname{dim} F_{n}=+\infty$ for all $n \in I, I \subseteq \mathbf{N}$ infinite. Without loss of generality $I=\mathbf{N}$. Let

$$
\widehat{\widehat{F}}=\frac{\ell^{\infty}\left(F_{n}\right)}{C_{\mathcal{U}}\left(F_{n}\right)}=\left\{\left(\widehat{v}_{n}\right)_{u}: \widehat{v}_{n} \in F_{n} \quad \forall n\right\}
$$

Then $\operatorname{dim} \hat{\hat{F}}=\infty\left([5]\right.$, Theorem 3.1). Let $\widehat{\hat{v}} \in \hat{\widehat{F}}, \widehat{\widehat{v}}=\left(\hat{v}_{n}\right)_{u} \neq 0$ and $\widehat{v}_{n} \in F_{n}$. Hence $\widehat{T}_{n} \widehat{v}_{n}=\lambda_{n} \widehat{v}_{n}$. Since $\left\|\widehat{T}_{n}^{k+1}-\widehat{T} \widehat{T}_{n}^{k}\right\| \longrightarrow 0$ it follows that

$$
\lambda^{k+1} \widehat{\hat{v}}=\left(\lambda_{n}^{k+1} \widehat{v}_{n}\right)_{u}=\left(\widehat{T} \widehat{T}_{n}^{k} \widehat{v}_{n}\right)_{u}=\lambda^{k} \widehat{\widetilde{T}} \widehat{\hat{v}} .
$$

But $\lambda \neq 0$, hence $\widehat{\widehat{T}} \widehat{\hat{v}}=\lambda \widehat{\widehat{v}}$. Thus $\widehat{\widehat{F}} \subseteq \operatorname{Ker}(\lambda-\widehat{\widehat{T}})$. Then $\operatorname{dim} \operatorname{Ker}(\lambda-\widehat{\widehat{T}})=+\infty$. By Theorem 2.1, $\lambda$ cannot be a Riesz point of $\sigma(T)$. This contradiction proves that for some $n_{0} \in \mathbf{N}$ and all $n \geq n_{0}$ $\operatorname{dim} \operatorname{Ker}\left(\lambda-\widehat{T}_{n}\right)<+\infty$. Again by Theorem $2.1 \forall n \geq n_{0}, \lambda_{n}$ is a Riesz point of $\sigma\left(T_{n}\right)$.

Lemma 3.2. Let $T, T_{n}, n=1,2, \ldots$ and $\lambda$ be as Theorem 2.2. Then, there exist $n_{0} \in \mathbf{N}, \rho^{*}>0$ such that for all $n \geq n_{0}, \sigma\left(T_{n}\right) \cap$ $B\left(\lambda, \rho^{*}\right)$ consists of a finite number of Riesz points of $\sigma\left(T_{n}\right)$, which do not accumulate on $\partial B\left(\lambda, \rho^{*}\right)$. 
Proof. Since $\lambda \in \partial_{\infty} \sigma(T)$ is a Riesz point of $\sigma(T)$, there exists a curve $\gamma:[0,+\infty] \longrightarrow \mathcal{C}$ such that $\gamma(0)=\lambda, \gamma(+\infty)=\infty$ and $\gamma([0,+\infty[) \subseteq \rho(T)$. For each $\delta>0$ let

$$
V_{\delta}:=\{z \in \mathcal{C}: \operatorname{dist}(z, \gamma(] 0,+\infty[))<\delta\}
$$

Notice that for some $\delta_{0}>0, \bar{V}_{\delta_{0}} \cap \sigma(T)=\{\lambda\}$. Write $V=V_{\delta_{0}}$. Let $\rho>0$ and let $V_{1 \rho}:=B(\lambda, \rho) \cap V$ and $V_{2 \rho}$ be the complement of $V_{1 \rho}$ in $V$. Then $V=V_{1 \rho} \cup V_{2 \rho}$. Without loss of generality, we suppose that $0 \notin V$. The proof proceeds in a series of claims. $V_{2 \rho}=\emptyset$.

Claim 1. $\forall \rho>0, \rho<\delta_{0}, \exists n_{\rho}^{*} \in \mathbf{N}$ such that $\forall n \geq n_{\rho}^{*}, \sigma\left(T_{n}\right) \cap$

Otherwise, there exists $\rho>0, \rho<\delta_{0}$ such that for each $n \in$ $\mathbf{N} \exists n^{\prime} \geq n$ and a point $\lambda_{n^{\prime}} \in \sigma\left(T_{n^{\prime}}\right) \cap V_{2 \rho}$. Since $T_{n^{\prime}}$ is uniformly bounded, $\left\{\lambda_{n^{\prime}}\right\}$ is a bounded sequence. Call $\left\{\lambda_{n^{\prime}}\right\}$ again as $\left\{\lambda_{n}\right\}$. Without loss of generality we suppose that $\lambda_{n} \longrightarrow \mu \in \bar{V}_{2 \rho}$. Now it is easy to check that $\mu \in \sigma(T)$. In fact we can take $\lambda_{n} \in \partial_{\infty} \sigma\left(T_{n}\right)$. Hence $\widehat{T}_{n} \widehat{x}_{n}=\lambda_{n} \widehat{x}_{n}$ for some $\widehat{x}_{n} \in \widehat{X},\left\|\widehat{x}_{n}\right\|=1$. Then

$$
\lambda_{n}^{k+1} \widehat{x}_{n}=\widehat{T}_{n}^{k+1} \widehat{x}_{n}=\widehat{T} \widehat{T}_{n}^{k} \widehat{x}_{n}=\lambda_{n}^{k} \widehat{T}_{n} \widehat{x}_{n}
$$

Thus $\widehat{T}_{n} \widehat{x}_{n}=\lambda_{n} \widehat{x}_{n}$. Hence $\widehat{\widehat{T}}\left(\widehat{x}_{n}\right)_{u}=\mu\left(\widehat{x}_{n}\right)_{\mathcal{u}}$. Therefore $\mu \in \sigma(\widehat{\widehat{T}})=$ $\sigma(\widehat{T})$, a contradiction since $\bar{V}_{2 \rho} \cap \sigma(T)=\emptyset$.

Claim 2. $\exists \rho \in] 0, \rho_{0}\left[, \exists n_{0} \in \mathrm{N}\right.$ such that $\forall n \geq n_{0}, \sigma\left(T_{n}\right) \cap B(\lambda, \rho)$ contains only a finite number of points.

Otherwise: $\forall \rho \in] 0, \rho_{0}\left[, \forall n \in \mathrm{N}, \exists n^{\prime} \geq n\right.$ such that $\sigma\left(T_{n^{\prime}}\right) \cap B(\lambda, \rho)$ contains an infinite number of points. Using claim 1 , we construct sequences $\rho_{i}, n_{i}$, such that $\rho_{i+1}<\rho_{i}, \rho_{i} \longrightarrow 0, n_{i}<n_{i+1}, n_{i} \longrightarrow+\infty$ such that

- $\sigma\left(T_{n_{i}}\right) \cap B\left(\lambda, \rho_{i}\right)$ contains an infinite number of points and

- $\sigma\left(T_{n}\right) \cap V_{2 \rho_{i}}=\emptyset$ for all $n \geq n_{i}$

Notice that this implies that the approximated point spectrum of $T_{n_{i}}$ in $B\left(\lambda, \rho_{i}\right)\left(\equiv A \sigma\left(T_{n_{i}}\right) \cap B\left(\lambda, \rho_{i}\right)\right)$ contains an infinite number of 
points. Let $\lambda_{n_{i}} \in A \sigma\left(T_{n_{i}}\right) \cap B\left(\lambda, \rho_{i}\right)$ be an accumulation point. Then, $\operatorname{dim} \operatorname{Ker}\left(\lambda_{n_{i}}-\widehat{T}_{n_{i}}\right)=\infty$ (Theorem 2.1 above). Let $F_{n_{i}}=\operatorname{Ker}\left(\lambda_{n_{i}}-\widehat{T}_{n_{i}}\right)$. As usual we prove that

$$
\widehat{\widehat{F}}=\frac{\ell^{\infty}\left(F_{n_{i}}\right)}{C_{\mathcal{U}}\left(F_{n_{i}}\right)} \subseteq K \operatorname{Rer}(\lambda-\widehat{\widehat{T}}) .
$$

Hence

$$
\operatorname{dim} \operatorname{Ker}(\lambda-\hat{\widehat{T}})=\infty,
$$

a contradiction. Thus, claim 2 is proved.

Claim 3. $\exists \rho^{*}>0\left(\rho^{*}<\rho\right), \exists n_{0}^{*} \in \mathrm{N}, n_{0}^{*} \geq n_{0}$ such that $\forall n \geq$ $n_{0}^{*}, \sigma\left(T_{n}\right) \cap B\left(\lambda, \rho^{*}\right)$ contains only a finite number of Riesz points of $\sigma\left(T_{n}\right)$.

Otherwise: $\forall \rho^{*}>0\left(\rho^{*}<\rho\right), \forall n_{0}^{*} \geq n_{0}$ exists $n^{\prime} \geq n_{0}^{*}$ such that $\sigma\left(T_{n^{\prime}}\right) \cap B\left(\lambda, \rho^{*}\right)$ contains points which are not Riesz points of $\sigma\left(T_{n^{\prime}}\right)$. Inductively and using claims 1 and 2 above we construct sequences $\rho_{n_{i}}^{*} \downarrow 0, n_{i} \uparrow+\infty, \lambda_{n_{i}} \in \partial_{\infty} \sigma\left(T_{n_{i}}\right) \cap B\left(\lambda, \rho_{n_{i}}^{*}\right)$ where $\lambda_{n_{i}}$ is not a Riesz point of $\sigma\left(T_{n_{i}}\right)$. This contradicts Lemma 3.1.

Finally, let $\lambda_{n} \in \sigma\left(T_{n}\right) \cap B\left(\lambda, \rho^{*}\right), \lambda_{n} \longrightarrow \mu$. Without loss of generality $\rho^{*}$ is such that $\sigma(T) \cap B\left(\lambda, \rho^{*}\right)=\{\lambda\}$. Now, it is easy to prove that $\mu=\lambda$. The lemma is proved.

Remark. To prove Theorem 2.2 the full strength of Lemma 3.2 above is not needed. In spite of it, we consider it interesting in itself to be included here.

Finally let us state without proof the well known lemma:

Lemma 3.3. ([3] Proposition 2.1) Let $T \in \mathcal{L}(X)$ be such that for some constant $M>0$

$$
\|(z-T) x\| \geq M\|x\| \quad \forall x \in X, \forall z \in K
$$


where $K$ is compact subset of $C$. Let $T_{n} \in \mathcal{L}(X)$ be such that for some $k \in \mathbf{N},\left\|\left(T-T_{n}\right) T_{n}^{k}\right\| \longrightarrow 0$. Then, $\exists M_{1}>0$ and $n_{0} \in \mathbf{N}$ such that

$$
\left\|\left(z-T_{n}\right) x\right\| \geq M_{1}\|x\| \quad \forall x \in X, \forall z \in K, \forall n \geq n_{0} .
$$

Proof of Theorem 2.2. Being $\Gamma=\partial B\left(\lambda, \rho^{*}\right)$, with $\rho^{*}$ as in Lemma 3.2, let us prove that $T_{n}-z \stackrel{s}{\longrightarrow} T-z, \forall z \in \Gamma$. Then, Proposition 3.1 will do the rest. The only thing to be checked is that for any $z \in$ $\Gamma$, there exists $M(z)>0, n_{0}(z) \in \mathbf{N}$ such that $R\left(z, T_{n}\right)$ exists and $\left\|R\left(z, T_{n}\right)\right\| \leq M(z), \forall n \geq n_{0}(z)$. But this is inmediate in view of Lemmas 3.2 and 3.3 above.

\section{References}

[1] Ahues, M.: A class of strongly stable operator approximations. J. Austr. Math. Soc. Ser. B 88 (1987), 435-442.

[2] Anselone, P.M.: Collectively Compact Operator Approximation Theory. (Prentice-Hall, Englewood Cliffs, New Jersey, 1971).

[3] Asadzahed, M.: $L_{p}$ and eigenvalue error estimates for discrete ordinates method for two-dimensional neutron transport. SIAM. J. Numer. Anal. 26 (1989), 66-87.

[4] Bouldin, R.: Operator Approximations with stable eigenvalues. J. Austr. Math. Soc. Ser. A., 49 (1990), 250-260.

[5] Boyd, M.W. and Transue, W.R.: Properties of ultraproducts. (Rendiconti del Circolo Matemático di Palermo, Serie II, Tomo XXVIII, 387 397,1979 ).

[6] Caselles, V.: On the periphenal spectrum of positive operators, Israel J. of Math. 58 (1986), 144-160.

[7] Chatelin, F.:Spectral approximation of linear operators (Academic Press, New York, 1983).

[8] Kato, T.: Perturbation theory for linear operators (Springer-Verlag, 1982). 
[9] Schaefer, H.H.: Banach lattices and positive operators (SpringerVerlag, 1974).

[10] Thambar Nair, M.: On strongly stable approximations, J. Austr. Math. Soc. Ser. A., 52 (1992), 251-260.

Departament de Matemàtica Aplicada

j Astronomia

Universitat de València

Dr. Moliner, 50

46100 Burjassot (Valencia)

SPAIN.
Departament de Matematiques

$i$ informatica

Universitat de les Illes Balears

Ctra. Valldemossa, $\mathrm{Km} \mathrm{7,5}$

07071 Palma de Mallorca (Balears)

SPAIN.

Recibido: 17 de marzo de 1993

Revisado: 30 de septiembre de 1993 